\title{
SOME PRELIMINARY OBSERVATIONS ON THE EARLY RECEPTOR POTENTIAL OF THE ISOLATED FROG RETINA
}

\author{
Isao HanAwA*, Katsuhiko MATSUMURA \\ AND Tetsuji MATsUURA \\ Department of Physiology, Osaka City University Medical School, \\ Abeno-ku, Osaka, Japan
}

The early receptor potential (early RP), which results from very intense photo-stimulation of the vertebrate retina, was first described by BROWN and MuraKami (1964a, b). From the evidence discovered in the last few years we know that its latency is of the order of several ten microseconds, that its action spectrum agrees with that of rhodopsin, that its amplitude is a linear function of light intensity, that it persists when the retina is placed in grossly abnormal chemical environment, and that it persists when the retina is frozen (Cone, 1964 ; PAK and Cone, 1964; ARDEN and IKEDA, 1965 ; Brown, 1965 ; BROWN, Watanabe and Murakami, 1965; Cone, 1965; PAK, 1965; PAK and Ebrey, 1965 ; BRINDLEY and GARDNER-MEDwin, 1966), it has been supposed that the early RP represents perhaps a direct manifestation of changes in visual pigments due to absorption of light, but the mechanism to elicit this response is still unknown. The recent report of Cone (1967) that the early RP is generated as rhodopsin proceeds through the initial stages of bleaching intermediates of rhodopsin would seem to support the concept that the responses are generated by the net displacements of charge in the pigment molecule.

The experiments reported here are a part of series of studies on the early $\mathrm{RP}$ of the isolated frog retina, and an attempt was made to know some fundamental properties of the early RP; such as effects of successive light flashes, effects of potassium ions and effects of polarization; by using the method which has been described by Hanawa, KugE and Matsumura (1967). This method is particularly advantageous for the investigation, since it serves to eliminate the photovolatic artifacts which have been produced by the presence of metal electrodes, and the chemical environments on the scleral or vitreous side of the retina are easily exchangeable independently.

Received for publication November 30, 1967

塙 功, 松村克彦, 松浦哲志

* Present address: Department of Physiology, Gifu University School of Medicine, Gifu, Japan 


\section{METHOD}

Preparation of the isolated tissue. Experimental animals in this study were darkadapted bullfrogs (R. catesbiana). Following three types of preparations were made by dissection under dim white light; 1) the retina with the pigment epithelium, 2) the retina deprived of the pigment epithelium, 3) the pigment epithelium-choroid complex. Details of making the retina with the pigment epithelium and that without it have been described previously (Hanawa, Kuge and Matsumura, 1967). To make the pigment epithelium-choroid complex, the eye-ball from which the sclera was removed was placed on a sheet of filter paper having a small hole (diameter $6 \mathrm{~mm}$ ) with the corneal side upwards. Then the posterior half of the eye-ball was dissected away from the anterior portion along the equator. A sheet of filter paper was then placed on the retina. After a few moments the filter paper adhered to the retina and was stripped off gently from the choroid, thus the retina was removed entirely. The preparation was used as a membrane to separate the solutions in the experimental apparatus.

Experimental apparatus. The early RP was measured in the apparatus as shown diagrammatically in FIG. 1. The experimental apparatus consisted of two compartments made of acryl resin plates, with a small circular hole (diameter $6 \mathrm{~mm}$ ) on the separating plane. A tissue surface area of $28.3 \mathrm{~mm}^{2}$ was exposed to the both $8 \mathrm{ml}$ of solution. The bathing solution was stirred throughout the experiment by a stream of pure oxygen or nitrogen bubbles saturated with vapor. Two narrow agar bridges were prepared by

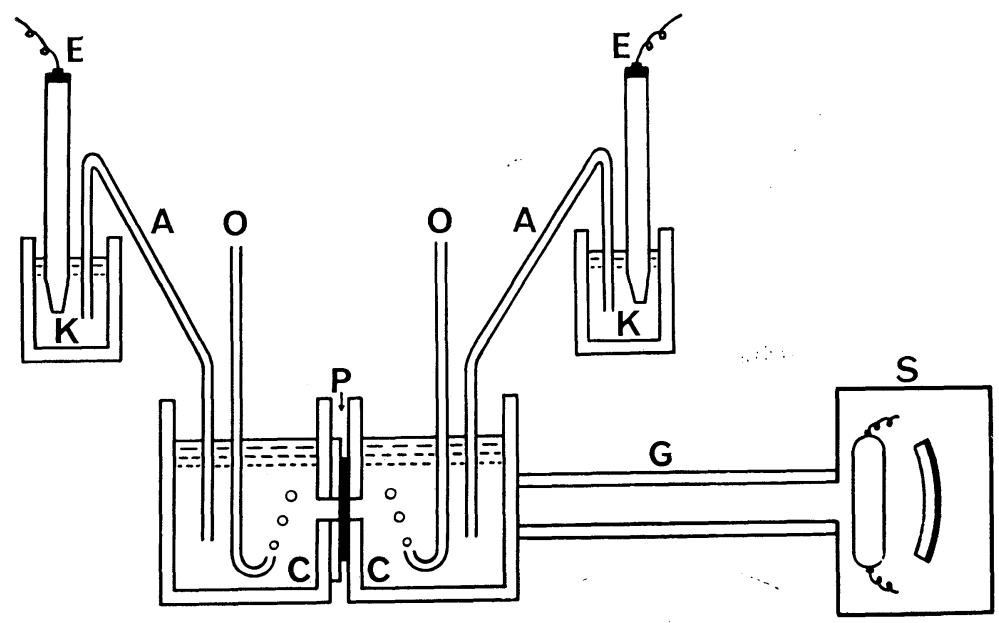

FIG. 1. Diagram of apparatus.

C : Compartment that was made of acryl resin plates, containing $8 \mathrm{ml}$ of bathing solution on each side of the retina.

P : Isolated preparation.

$\mathrm{O}$ : Inlets for oxygen or nitrogen gas.

A : Agar bridge connecting scleral side or vitreous side solution with the calomel electrode.

E : Calomel electrode.

$\mathrm{K}$ : Saturated $\mathrm{KCl}$ solution.

$\mathrm{S}$ : Commercial photographic strobe lamp.

$\mathrm{G}$ : Glass cylinder that was covered with aluminum foil. 
4 per cent agar and the bathing solution opened on either compartment. The other end of each agar bridge made contact with saturated $\mathrm{KCl}$-calomel electrodes connecting to a cathode ray oscilloscope through a $\mathrm{RC}$ coupled pre-amplifier (time constant $1 \mathrm{sec}$ ). The pre-amplifier and cathode ray oscilloscope recorded the difference of electrical potential between the solution on the scleral side and that on the vitreous side of the preparation. To minimize photovolatic artifacts, the calomel electrodes were shielded from the light as much as possible. The polarity of all records was such that an upward deflexion indicates positivity of the vitreous side of the preparation. All experiments were carried out at room temperature between 20 and $25^{\circ} \mathrm{C}$.

Light source. For measurements of the early RP, the stimulus flashes were produced by a commercial photographic strobe lamp (Kakonet Special, Kako Co., Japan) which gave an intense flash having an effective duration of $0.7 \mathrm{msec}$. To minimize electrical artifacts, the strobe lamp was enclosed in a soft-steel box, and the flash light was led to the preparation by a glass cylinder, $3.5 \mathrm{~cm}$ in diameter and $53 \mathrm{~cm}$ long, that was covered with aluminum foil, one end of which was placed close to the flash tube, and the other end was placed close to the experimental apparatus. All the preparations were illuminated by an intense flash from the vitreous side. Usually, no filter was placed in the stimulus beam. In some case, the retina was exposed to intense flashes through a cutoff filter (V-Y 50, Toshiba) that eliminated all wavelengths shorter than $500 \mathrm{~m} \mu$.

Polarizing current. To observe the effectiveness of the polarizing current on light responses, a dc current of $200 \mu \mathrm{A}$ was applied across the preparation through a pair of $\mathrm{Zn}-\mathrm{ZnSO}_{4}$ non-polarizable electrodes. The polarizing electrodes were placed in separate chambers connected to each compartment by a saline-agar bridge.

Bathing solution. The bathing solution mostly used for this experiment had the following composition (mM): $\mathrm{NaCl}, 72.0 ; \mathrm{KCl}, 2.5 ; \mathrm{MgSO}_{4}, 1.2 ; \mathrm{Na}_{2} \mathrm{SO}_{4}, 0.6 ; \mathrm{NaHCO}_{3}$, 25.0 ; $\mathrm{NaH}_{2} \mathrm{PO}_{4}, 3.0$; calcium gluconate, 0.9 ; glucose, 26.0. The $74.5 \mathrm{mM}$ potassium solution was prepared by replacing all the sodium chloride in the bathing solution with potassium chloride. All solutions were buffered with bicarbonate and phosphate at $\mathrm{pH}$ 7.5.

\section{RESULTS}

The effects of successive light flashes. Since the time of KüHNE (1878), it has been well known that the regeneration of rhodopsin in the isolated retina alone is slow and incomplete, but becomes faster and more complete if the retina is placed in contact with the pigment epithelium. Recently, CoNE (1964) reported that the amplitude of the early RP is linearly proportional to the amount of visual pigments bleached by a flash. Therefore, an attempt was carried out to confirm such augmentative activities of the pigment epithelium by measuring the early RP from the retina with the pigment epithelium intact or that of the retina alone. Both were immersed in a bathing solution stirred by a stream of oxygen or nitrogen bubbles, since the amplitude of the early $\mathrm{RP}$ would be related to the concentration of visual pigments in the outer segments if the responses are elicited by flashes of fixed intensity. All the preparation had been initially immersed for $30 \mathrm{~min}$ in the solution saturated with a given pure gas, and then stimulated by successive intense light flashes at 5 min intervals. The amplitude of the early $\mathrm{RP}$ was measured from the peak of the cornea-positive phase to that of the cornea-negative one. In all four 

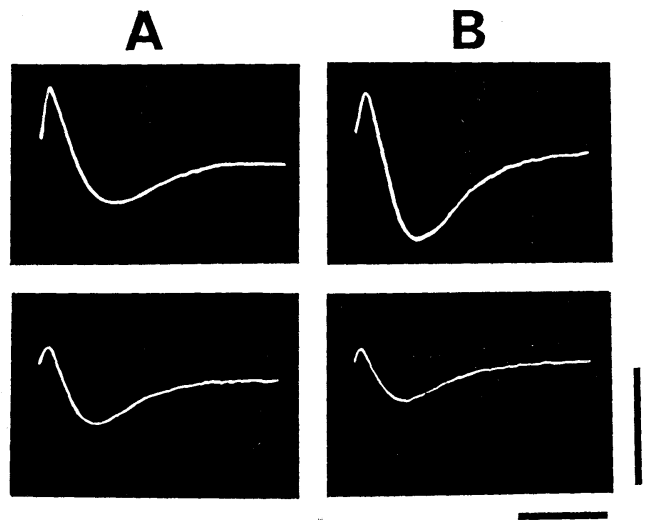

FIG. 2. Early RPs under four different conditions. Column $\mathrm{A}$ and $\mathrm{B}$ show responses from retinas in the oxygenated and the anaerobic conditions respectively. In each column, upper record is the response from the retina deprived of the pigment epithelium, and lower record is that from the retina with the pigment epithelium intact. All records are the first response. Voltage calibration: $0.5 \mathrm{mV}$. Time : $2 \mathrm{msec}$.

cases, every early RP was a biphasic response consisting of an initial corneapositive phase followed by a slower cornea-negative phase, as it has been found in a number of other vertebrate species. Typical examples of the first response in four cases are shown in FIG. 2. Regardless of the bubbling gas, the amplitude of response was $0.3-0.8 \mathrm{mV}$ and the ratio of heights of positive and negative phases was $0.3-0.7$ in a good preparation. However, under given experimental conditions, larger signals were obtained from the retina alone than from the retina with the pigment epithelium intact, and the amplitude maximum of the negative phase was reached somewhat earlier in the retina with the pigment epithelium than that of the retina alone. This difference in peak latency may be due to contamination by a rapid response from the pigment epithelium-choroid complex as found by BROWN (1965). In fact, a small photostable response similar to the second phase described by BROWN and GAGE (1966a, b) could be obtained at our stimulus intensity from the pigment epithelium-choroid complex of a frog's eye (FIG. 7Db).

The decay of early RP by successive light flashes at 5 min intervals is shown in FIG. 3. The amplitude of response was expressed as a percentage of that of the first response. In the case of the retina alone, a roughly linear relationship between the $\log$ of response amplitude and the flash number was obtained in either the oxygenated and the anaerobic conditions. On the other hand, the responses of the retina with the pigment epithelium were more resistant to light adaptation than that of the retina alone and they were reduced much more readily in the anaerobic than in the oxygenated condition. On the 7th flash, the response amplitude decreased to $74.0 \pm 6.8$ per cent of its initial value in the oxygenated and to $50.2 \pm 4.4$ per cent in the anaerobic condition. Regardless of reduction in the response amplitude by successive light flashes, peak latencies of the positive and negative phases remained unchangeable in all four cases. The negative phase tends to decay more rapidly, however, than the positive phase. In the case of the retina alone, the ratio of 

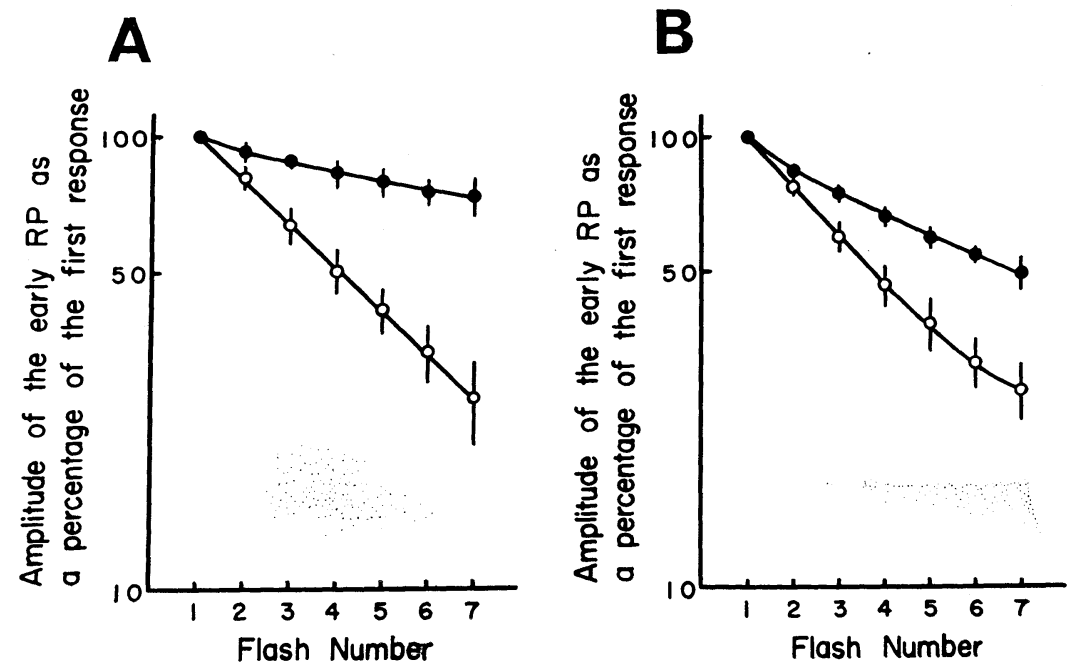

FIG. 3. The effects of successive light flashes on the amplitude of the early RP. $A:$ in the oxygenated condition, B: in the anaerobic condition. In each figure, shows the amplitude of the response from the retina with the pigment epithelium intact, $\bigcirc$ shows that from the retina deprived of the pigment epithelium. The abscissa is the flash number. Successive flashes were given at $5 \mathrm{~min}$ intervals. The ordinate is the amplitude of the early RP which is expressed as a percentage of that of the first response.

heights of positive and negative phase at the 7th flash slightly increased to $0.67 \pm 0.16$ from its initial value of $0.53 \pm 0.05$.

These facts indicate that the pigment epithelium is necessary for the sufficient regeneration of the visual pigments, and it requires oxygen to attain maximal activities. In the absence of oxygen, the amplitude of the early RP decreased to 68 per cent of that in the presence of oxygen on the 7 th flash. This is in good agreement with the fact that the regeneration of rhodopsin declined to 63 per cent of the control by clamping the ocular artery of the frog's eye (Kinoshita, Nakano, Sugimoto and Hosoya, 1960).

The effects of increasing the potassium concentration. The effects of increasing the potassium ion concentration in the bathing solution have been reported recently by PAK (1965) and PAK, ROZZI and EBREY (1967). They suggested that the negative phase of the early RP is preferentially enhanced in the isotonic potassium chloride, and the amount of enhancement appears too large to be ascribed to the small difference in conductivity of the test solution. We have performed similar experiments, the results of which are shown in Figs. 4 and 5. The first response was measured with the retina deprived of the pigment epithelium which had been immersed in the oxygenated bathing solution containing $2.5 \mathrm{mM}$ potassium for $30 \mathrm{~min}$. The solutions on both sides of 


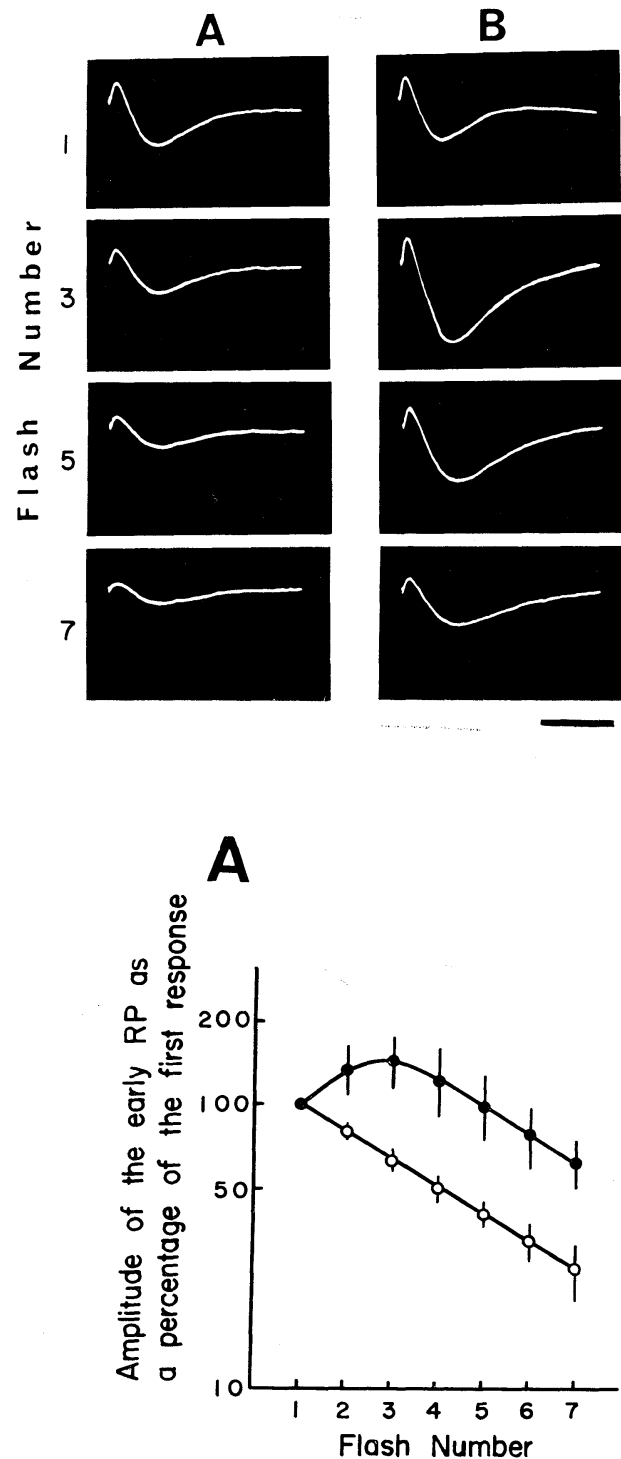

FIG. 4. The effects of increasing the potassium concentration in the bathing solution. Columns $\mathrm{A}$ and $\mathrm{B}$ show responses from retinas immersed in solutions containing 2.5 and $74.5 \mathrm{mM}$ potassium respectively. In each case, both surfaces of the retina were exposed to the test solution, and the retina was stimulated by successive light flashes at $5 \mathrm{~min}$ intervals. Voltage calibration : $0.5 \mathrm{mV}$. Time : $2 \mathrm{msec}$.

FIG. 5. The effects of increasing the potassium concentration in the bathing solution.

A : The effect of successive light flashes on the amplitude. The ordinate is the amplitude of the early RP which is expressed as a percentage of that of the first response.

B : The effect of successive light flashes on the ratio of heights of positive and negative phases.

In each figure, $\bigcirc$ and show the responses from retinas immersed in solutions containing 2.5 and $74.5 \mathrm{mM}$ potassium respectively. The retinas were stimulated by successive light flashes at 5 min intervals. 
the retina were then replaced by a solution containing $74.5 \mathrm{mM}$ potassium, and the following responses were recorded at $5 \mathrm{~min}$ intervals. The typical example of this experiment is shown in FIG. 4. When the potassium concentration in the bathing solution increased, both the positive and the negative phase were successively enhanced, then the amplitude reduced gradually. The amount of enhancement of the negative phase was larger than that of the positive phase. At the 4 th flash, therefore, the ratio of heights of positive and negative phase was decreased to $0.26 \pm 0.04$ from $0.52 \pm 0.10$ of the first response (FIG. $5 B$ ). In addition, the peak latency increased to $115-131$ per cent of that of the first response. In FIG. 5A the amplitude of the early RP, that was measured from the peak of the cornea-positive phase to that of the cornea-negative phase, was expressed as a percentage of that of the first response, and was plotted as functions of flash number. Each plotted point represents the mean of five measurements in different preparations. As compared with the control response that was obtained from the retina bathing in the solution containing $2.5 \mathrm{mM}$ potassium ions, it is interesting to note that the amplitude of the early RP was gradually increased with time and it reached the maximum increase of 2.5 -fold over the control value after 15 min following the application of the test solution, and then it decreased nearly parallel to that in the control solution. These results clearly indicate that on increasing the potassium concentration in the bathing solutions on both sides of the retina, the early RP is increased in amplitude and decreased in ratio of the heights of positive and negative
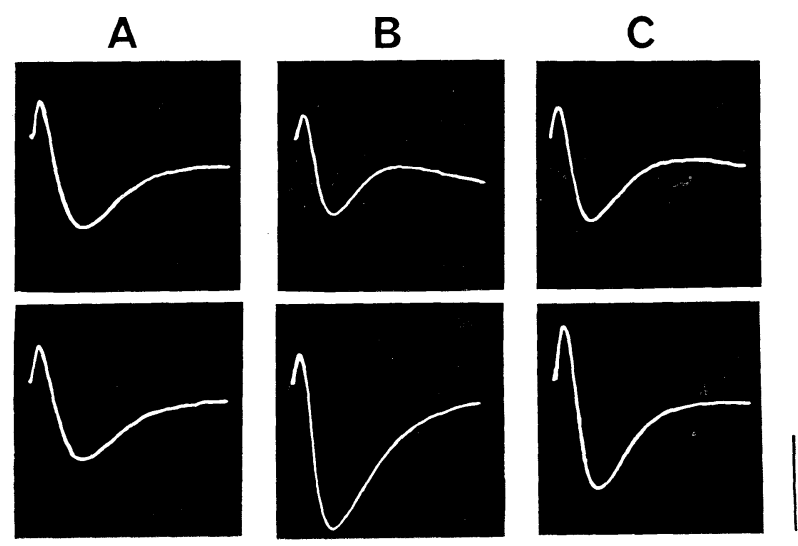

FIG. 6. The effects of increasing the potassium concentration on either side of the retina. In each column, upper record is the first response from the retina immersed in the solution containing $2.5 \mathrm{mM}$ potassium, and lower record is the second response from the retina after $30 \mathrm{~min}$ following an application of the test solution on either one side of the retina. (A : control, B : receptor side, C: vitreous side). Voltage calibration : $0.5 \mathrm{mV}$. Time: $2 \mathrm{msec}$. 
phases. Such a decrease in ratio was observed even if a cutoff filter eliminating all wavelengths shorter than $500 \mathrm{~m} \mu$ was used. It seems apparent, therefore, that a decrease in ratio does not simply depend on any inhibition of the photoregeneration of the visual pigments from intermediate, especially meta-rhodopsin II which develops a slow positive response, as described by CONE (1967) and PAK and BoEs (1967).

An enhancement of the response was also observed when the bathing solution on either side of the retina was replaced by a solution containing $74.5 \mathrm{mM}$ potassium. In the experiments shown in FIG. 6, the first responses in each column were recorded from the retina alone which had been immersed in the solution containing $2.5 \mathrm{mM}$ potassium, and the second responses were obtained after 30 min following an application of the test solution on either one side of the retina. Column A shows the responses under normal condition, and the second response decreased in amplitude to 89.0 per cent of the first

A

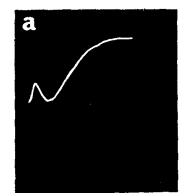

b
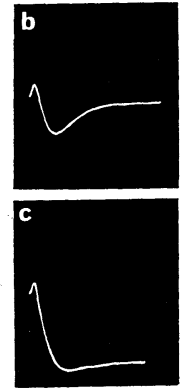

B
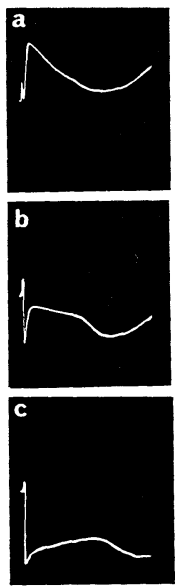

C
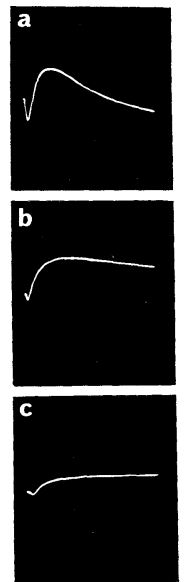

D

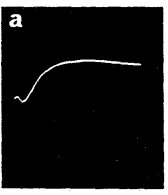

b
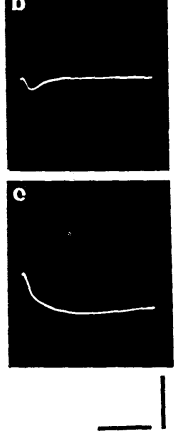

FIG. 7. The effects of polarization on the early RP. In each column, upper record is the response when the vitreous side of the preparation was polarized negative to the scleral side, middle record is a control where the preparation was not polarized, and lower record is that when the vitreous side of the preparation was polarized positive relative to the scleral side. Column A: the retina with the pigment epithelium intact; voltage calibration, $0.5 \mathrm{mV}$; time, $2 \mathrm{msec}$. B: the retina with pigment epithelium intact; voltage calibration, $0.5 \mathrm{mV}$; time, $20 \mathrm{msec}$. $\mathrm{C}$ : the retina with the pigment epithelium intact; voltage calibration, $2 \mathrm{mV}$; time, $50 \mathrm{msec}$. D: the pigment epithelium-choroid complex; voltage calibration, $0.5 \mathrm{mV}$; time, $2 \mathrm{msec}$. 
response. Exposing the vitreous surface to the test solution, the early RP increased in amplitude to 133.8 per cent of the first response, while there were no distinct changes on the peak latency and the ratio of heights of positive and negative phase. On the other hand, exposing the receptor side of the retina to the test solution, the early RP increased in amplitude to 168.2 per cent of the first response and decreased in ratio to the heights of positive and negative phases to 0.25 from 0.34 of the first response. In addition, the peak latency increased to 105-126 per cent of that of the first response.

The effects of polarization. In order to find out whether polarization affects the early RP, a dc current of $200 \mu \mathrm{A}$ was passed through the holes in the separating plates and the retina. In this experiment, the isolated retina with the pigment epithelium intact was immersed in the oxygenated bathing solution. When the vitreous side of the retina was polarized negative, relative to the scleral side, the positive phase of the early RP was slightly enhanced and the negative phase completely disappeared, and a slow positive-going hump following the positive phase was detected (FIG. 7Aa). In addition, it was found that the peak of this slow potential appeared between the positive phase of the early RP and the leading edge of the a-wave $(\mathrm{Ba})$. The peak latency was about $4 \mathrm{msec}$ for this slow potential, while the mid point of decline was about $32.5 \mathrm{msec}$. Under this condition, a decrease in the a-wave latency and an increase in the b-wave amplitude were also observed (Ca). On the other hand, when the vitreous side of the retina was polarized positive, relative to the scleral side, the early RP is slightly decreased in the amplitude of the positive phase and remarkably increased in that of the negative phase ( $\mathrm{Ac}$ and $\mathrm{Bc}$ ). Moreover, it was observed an increase in the a-wave latency and a decrease in the b-wave amplitude $(\mathrm{Cc})$.

The above findings have led us to two questions; 1 . whether or not the early RP is contaminated by a new slow response which is elicited by an intense flash when the retina is polarized, and 2. where is the origin of such a slow response? The following experiments were carried out to answer these two questions. Using the isolated retina deprived of the pigment epithelium, regardless of direction of polarizing current, the early RP of retinal photoreceptors did not change in its amplitude and shape. As shown in FIG. 7D, however, a slow response could be elicited from the pigment epithelium-choroid complex by an intense flash when a dc current was passed through the preparation. Under the control condition, only a rapid small negative reponse which is the second phase described by BROwn (1965) was detected (Db). When the vitreous side of the preparation was polarized negative, relative to the scleral side, a slow positive response following the negative phase of the early potential could be elicited by an intense flash, and the negative phase of the early potential decreased in amplitude (Da). By contrast, the negative phase became strikingly prolonged and enhanced by a current of the opposite direc- 
tion (Dc). These responses were highly resistant to light adaptation. These findings strongly suggest that the early RP of the retinal photoreceptors is not affected by a transretinal polarizing current, and that the slow response which is elicited by an intense flash under polarized conditions is generated from the pigment epithelium-choroid complex.

\section{DISCUSSION}

The effects of increasing the potassium concentration. As shown in the present study, increasing the potassium concentration in the bathing solution, the amplitude of the early RP is increased. In general, the following four possibilities would be considered as an explanation of an increase in amplitude of the early RP, if the response is generated by charge displacements in the visual pigment molecule ; 1) an increase in the transretinal conductivity including the electrical conductivity of the solution used, 2) an increase of rhodopsin concentration in the visual cells or an increase of the amount of rhodopsin molecules bleached by a flash, 3) an inhibition upon the photoregeneration of the visual pigments from intermediates in the bleaching of rhodopsin, since the opposite potential changes in polarity to the early RP are associated with photoregeneration of the visual pigment (ARDEN and IKEDA, 1965; ConE, 1967; PAK and BOES, 1967), 4) a decrease in the membrane resistance of the visual cells. As described in the preceding paper (HANAwA, KugE and Matsumura, 1967), the permeability of potassium ions through the retina proper is at least three times higher than those of sodium and chloride ions, and it has the same value in either direction. Therefore, an increase of the potassium concentration in the bathing solution on either one side of the retina leads to an increase in the transretinal electrical conductivity. The evidence in the present study, that the early RP is enhanced to about 140 per cent of the control by an increase of potassium concentration on the vitreous side, clearly indicates that an increase in the transretinal electrical conductivity leads to an enhancement of the response. In this case, it is interesting to note that the peak latency and the ratio of heights of positive and negative phase remained unchanged. The second and third possibilities would be excluded in the present problem, since it can be seen that the amplitude change, after passing through a maximum, in high potassium solutions is nearly parallel with that in the control solution (FIG. 5A), and that a decrease in ratio in high potassium solutions is not affected by an intense flash through a cutoff filter eliminating all wavelength shorter than $500 \mathrm{~m} \mu$. The fourth possibility is not so easy to explain, but the facts that increasing the potassium concentration on the receptor side caused the decrease in ratio and the increase in peak latency of negative phase clearly suggest that potassium ions affect some mechanisms underlying the early RP in the visual cells. More recently, SMITH and BROWN 
(1966) reported that a depolarizing potential of $0.5-1.5 \mathrm{mV}$ amplitude is evoked from invertebrate photoreceptors, retinular cells of horseshoe crab, by intense light stimulus by means of intracellular recordings. They observed that the amplitude and polarity of this response are a function of membrane potential. No similar response has yet been detected from the visual cell membrane of vertebrate eyes, but it seems likely that a depolarizing potential, which is a response of opposite polarity to the negative phase of the early RP, is elicited from the cell membrane of outer segment, since an application of alum to the retina produces a constant decrease in ratio of heights of positive and negative phase (Hanawa, Matsumura and Matsuura, 1968). Thus, it is possible to explain the decrease in ratio of heights of positive and negative phase in high potassium solutions by assuming a decrease in amplitude of a depolarizing potential from the cell membrane of the outer segment.

The effects of polarization. When a pigment epithelium-choroid complex containing melanin granules is stimulated by a high intensity light flash, it generates a fast electrical response similar to the early RP of retinal photoreceptors (BROWN, 1965). Since the response is photostable and has a spectral response curve which is essentially flat through the major portion of the visible range, the response appears to be mediated primarily by melanin (BROWN and CRAWFORD, 1967; EBREY and CONE, 1967). Under normal conditions this lightevoked response consists of three phases which seem comparable to the biphasic early RP and late RP of the retinal photoreceptors, and the second phase normally masks the first phase (BROWN and GAGE, 1966a, b). More recently, BROWN and CRAWFORD (1967) have shown that all three phases of the response are generated by the pigment epithelium cells, with no detectable contribution from choroidal cells, from the experiments using the microelectrode technique. As shown in the present study, when the vitreous side of the pigment epithelium-choroid complex is polarized negative relative, to the scleral side, a new slow positive response following the negative phase (BROwN's second phase) of the response is elicited by an intense light flash. The peak of this slow response appears between the positive phase of early RP and the leading edge of the a-wave. Under normal conditions a similar electrical response has been observed in pigeons, cats, albino rats and tortoises (YONEMURA and KAWASAKI, 1967). By contrast, when the vitreous side of the preparation is polarized positive to the scleral side, the negative phase of the response becomes strikingly prolonged and enhanced. In this case, the negative phase of the response is probably contaminated by a new slow negative response. Although these new slow responses which may correspond with the BROwN's third phase, have not definitely been established yet, we have observed a difference between both responses. BROWN and GAGE (1966a, b) suggested that the third phase is dependent on an active transport of sodium ions, since the response is reduced or abolished by a depletion of sodium ions or an addition of ouabain 
in the external medium. On the new response, however, the underlying process depends little upon the sodium ion concentrations in the bathing solution, since the response amplitude is little affected by the replacement of sodium chloride in the bathing solution with choline chloride or potassium chloride (HANAWA and MATSUMURA, unpublished observations). The details on this potential will be published later.

\section{SUMMARY}

1. The early receptor potential (early RP), which results from very intense photo-stimulation of the vertebrate eye, has been investigated in isolated preparations from the bullfrog's eye. All the preparations were placed as a septum separating the solutions in the apparatus.

2. The early RP of the retina with the pigment epithelium intact was more resistant to light adaptation than that of the retina alone, and its amplitude declined much more readily in the anaerobic than in the oxygenated condition. This clearly suggests that the pigment epithelium is necessary for the fast and sufficient regeneration of the visual pigments and that it requires oxygen to attain maximal activities.

3. Increasing the potassium concentration on both sides or the scleral side of the retina alone, the amplitude of the early $\mathrm{RP}$ was enhanced and the ratio of heights of positive and negative phases was decreased, whereas on the vitreous side the amplitude was increased, but the ratio remained unchanged. On the basis of these results it is proposed that an enhancement of the response is due to an increase of the transretinal electrical conductivity and a decrease in amplitude of a depolarizing potential which is probably elicited from the cell membrane of outer segments.

4. The early RP of retinal photoreceptors was not affected by a dc current across the retina, but the pigment epithelium-choroid complex generated a new slow response by an intense light flash under polarized condition.

The authors are indebted to Prof. E. KIMURA for his constant encouragement during the course of this investigation and for reading this manuscript. This study was supported by a Grant for Scientific Research from the Ministry of Education of Japan.

\section{REFERENCES}

1) ARden, G.B. ANd IKeda, H. (1965). A new property of the early receptor potenial of rat retina. Nature 208 : 1100-1101.

2) Brindley, G.S. And Gardner-Medwin, A.R. (1966). The origin of the early receptor potential of the retina. J. Physiol. 182: 185-194.

3) BRown, K.T. (1965). An early potential evoked by light from the pigment epithelium-choroid complex of the eye of the toad. Nature $207: 1249-1253$.

4) BROWN, K.T. AND CRAWFORD, J.M. (1967). Intracellular recording of rapid light- 
evoked responses from pigment epithelium cells of the frog eye. Vision Res. 7: 149-163.

5) Brown, K. T. And Gage, P.W. (1966a). Light-evoked potentials from the pigment epithelium-choroid complex of the toad's eye. Fed. Proc. 25:329.

6) BRown, K.T. And GaGe, P.W. (1966b). An earlier phase of the light-evoked electrical response from the pigment epithelium-choroid complex of the eye of the toad. Nature 211: 155-158.

7) Brown, K. T. ANd Murakami, M. (1964a). A new receptor potential of the monkey retina with no detectable latency. Nature 201: 626-628.

8) Brown, K. T. And Murakami, M. (1964b). Biphasic form of the early receptor potential of the monkey retina. Nature 204: 739-740.

9) Brown, K. T., Watanabe, K. and Murakami, M. (1965). Early and late receptor potentials of monkey cones and rods. Cold Spring Harb. Symp. quant. Biol. 30: 457-482.

10) Cone, R. A. (1964). Early receptor potential of the vertebrate retina. Nature 204 : 736-739.

11) Cone, R. A. (1965). The early receptor potential in the vertebrate eye. Cold Spring Harb. Symp. quant. Biol. 30: 483-490.

12) Cone, R.A. (1967). Early receptor potential: Photoreversible charge displacement in rhodopsin. Science 155: 1128-1131.

13) Ebrey, T.G. And Cone, R. A. (1967). Melanin, a possible pigment for the photostable electrical responses of the eye. Nature 213:360-362.

14) Hanawa, I., Kuge, K. and Matsumura, K. (1967). Effects of some common ions on the transretinal $\mathrm{dc}$ potential and the electroretinogram of the isolated frog retina. Jap. J. Physiol. 17: 1-20.

15) Hanawa, I., Matsumura, K. and Matsuura, T. (1968). A new property of the early receptor potential of the isolated frog eye. Jap. J. Physiol. 18:655-664.

16) Kinoshita, Y., Nakano, H., Sugimoto, N. And Hosoya, Y. (1960). Studies on the relation between the lipotropic substances and their transmethylation upon the rhodopsin synthesis in vivo. Osaka City Med. J. 6:17-24.

17) Kühne, W. (1878). Zur Photochemie der Netzhaut. Untersuchungen. physiol. Inst. Univ. Heidelberg. 1: 1-14.

18) PAK, W.L. (1965). Some properties of the early electrical response in the vertebrate retina. Cold Spring Harb. Symp. quant. Biol. 30: 493-499.

19) PAK, W.L. ANd BoEs, R. J. (1967). Rhodopsin: Responses from transient intermediates formed during its bleaching. Science 155: 1131-1133.

20) PAK, W. L. AND Cone, R. A. (1964). Isolation and identification of the initial peak of the early receptor potential. Nature $204: 836-838$.

21) PAK, W. L. ANd Ebrey, T. G. (1965). Visual receptor potential observed at sub-zoro temperature. Nature 205: 484-486.

22) PAK, W. L. ANd EBrey, T.G. (1966). Early receptor potentials of rods and cones in rodents. J. Gen. Physiol. $49: 1199-1208$.

22) Pak, W.L., Rozzi, V.P. And Ebrey, T.G. (1967). Effect of changes in the chemical environment of the retina on the two components of the early receptor potential. Nature 214: 109-110.

24) Smith, T. G. And Brown, J. E. (1966). A photoelectric potential in invertebrate cells. Nature 212: 1217-1219.

25) Yonemura, D. And Kawasaki, K. (1967). The early receptor potential in the human electroretinogram. Jap. J. Physiol. 17: 235-244. 